\title{
JORDAN FORMS AND NTH ORDER LINEAR RECURRENCES
}

\author{
THOMAS MCKENZIE, SHANNON OVERBAY, AND ROBERT RAY
}

\begin{abstract}
Let $p$ be a prime number with $p \neq 2$. We consider sequences generated by $n$th order linear recurrence relations over the finite field $Z_{p}$. In the first part of this paper we generalize some of the ideas in [6] to $n$th order linear recurrences. We then consider the case where the characteristic polynomial of the recurrence has one root in $Z_{p}$ of multiplicity $n$. In this case, we show that the corresponding recurrence can be generated by a relatively simple matrix.
\end{abstract}

\section{INTRODUCTION}

Let $p>2$ be a prime number, $n>1$ be an integer, and let

$$
S_{i}=a_{0} S_{i-n}+a_{1} S_{i-(n-1)}+\cdots+a_{n-1} S_{i-1}
$$

be an $n$th order linear recurrence with $a_{0}, \ldots, a_{n-1} \in Z_{p}$ and $a_{0} \neq 0$. A very nice discussion of such recurrences can be found in [5]. Since $\left(Z_{p}\right)^{n}$ has a finite number of elements, it is clear that any such $n$th order linear recurrence with initial conditions $S_{0}, S_{1}, \ldots, S_{n-1} \in Z_{p}$ will eventually repeat itself. The sequence is called uniformly distributed if each element of $Z_{p}$ appears the same number of times within these repeated periods.

Example 1.1. Consider the recurrence defined by $S_{0}=0, S_{1}=1, S_{2}=3$, and $S_{n}=3 S_{n-3}+3 S_{n-2}+S_{n-1}$, taken over $Z_{5}$. This generates the following uniformly distributed sequence:

$$
0,1,3,1,3,0,2,1,2,1,0,4,2,4,2,0,3,4,3,4 \text {. }
$$

Consider the $n \times n$ matrix

$$
A=\left[\begin{array}{ccccccc}
0 & 1 & 0 & 0 & \cdots & 0 & 0 \\
0 & 0 & 1 & 0 & \cdots & 0 & 0 \\
\cdot & \cdot & \cdot & \cdot & \cdots & \cdot & \cdot \\
. & . & . & . & \cdots & . & . \\
0 & 0 & 0 & 0 & \cdots & 1 & 0 \\
0 & 0 & 0 & 0 & \cdots & 0 & 1 \\
a_{0} & a_{1} & a_{2} & a_{3} & \cdots & a_{n-2} & a_{n-1}
\end{array}\right] .
$$




\section{JORDAN FORMS AND NTH ORDER LINEAR RECURRENCES}

The sequences defined by the recurrence relation

$$
S_{i}=a_{0} S_{i-n}+a_{1} S_{i-(n-1)}+\cdots+a_{n-1} S_{i-1}
$$

can be generated by the matrix relation

$$
\left[\begin{array}{c}
S_{i} \\
\cdot \\
\cdot \\
\cdot \\
S_{(n-1)+i}
\end{array}\right]=A^{i}\left[\begin{array}{c}
S_{0} \\
\cdot \\
\cdot \\
\cdot \\
S_{n-1}
\end{array}\right] .
$$

We note that $A$ is the companion matrix of the polynomial

$$
C(x)=\operatorname{det}(x I-A)=x^{n}-a_{n-1} x^{n-1}-\cdots-a_{1} x-a_{0}
$$

(see [3, p. 358]).

Since $a_{0} \neq 0, A$ is a unit in the ring of $n \times n$ matrices over $Z_{p}$ (i.e., $A \in G L_{n}\left(Z_{p}\right)$ ). Further, since this group of invertible $n \times n$ matrices is finite, $A$ generates a finite cyclic group of order $m$, for some natural number $m$. We will denote this group by

$$
G=\left\{A^{i} \mid 0 \leq i \leq m-1\right\} .
$$

Left multiplication of matrices on vectors defines a map from $G \times\left(Z_{p}\right)^{n}$ to $\left(Z_{p}\right)^{n}$. Since $A^{j}\left(A^{i} \mathbf{v}\right)=\left(A^{j} A^{i}\right) \mathbf{v},\left(Z_{p}\right)^{n}$ is a $G$-set (see [1, p. 176]). If a subset $U$ of $\left(Z_{p}\right)^{n}$ is closed under this action of $G$ and has the property that for all $\mathbf{u}^{\prime}, \mathbf{u} \in U$ there exists a $g \in G$ such that $g \mathbf{u}=\mathbf{u}^{\prime}$, then we call $U$ a transitive $G$-set. In other words, the transitive $G$-sets are just the orbits of the elements of $\left(Z_{p}\right)^{n}$ under repeated left multiplication by $A$.

\section{Transitive $G$-SETS}

If we select an arbitrary element $\mathbf{v}$ from $\left(Z_{p}\right)^{n}$, the orbit of $\mathbf{v}$ under the action of $G$ is the transitive $G$-set containing v. These transitive $G$-sets partition $\left(Z_{p}\right)^{n}$.

Example 2.1. Consider the sequence defined by $S_{n}=3 S_{n-3}+3 S_{n-2}+$ $S_{n-1}$, taken over $Z_{5}$. The action of the group generated by $A=\left[\begin{array}{lll}0 & 1 & 0 \\ 0 & 0 & 1 \\ 3 & 3 & 1\end{array}\right]$ partitions the $G$-set $\left(Z_{5}\right)^{3}$ into the following 8 transitive $G$-sets (orbits):

$$
H_{1}=\left\{\left[\begin{array}{l}
0 \\
0 \\
0
\end{array}\right]\right\}
$$

MISSOURI J. OF MATH. SCI., FALL 2014 
T. MCKENZIE, S. OVERBAY, AND R. RAY

$$
\begin{aligned}
& H_{2}=\left\{\left[\begin{array}{l}
0 \\
0 \\
1
\end{array}\right],\left[\begin{array}{l}
0 \\
1 \\
1
\end{array}\right],\left[\begin{array}{l}
1 \\
1 \\
4
\end{array}\right],\left[\begin{array}{l}
1 \\
4 \\
0
\end{array}\right],\left[\begin{array}{l}
4 \\
0 \\
0
\end{array}\right],\left[\begin{array}{l}
0 \\
0 \\
2
\end{array}\right],\left[\begin{array}{l}
0 \\
2 \\
2
\end{array}\right],\left[\begin{array}{l}
2 \\
2 \\
3
\end{array}\right],\left[\begin{array}{l}
2 \\
3 \\
0
\end{array}\right],\left[\begin{array}{l}
3 \\
0 \\
0
\end{array}\right],\right. \\
& \left.\left[\begin{array}{l}
0 \\
0 \\
4
\end{array}\right],\left[\begin{array}{l}
0 \\
4 \\
4
\end{array}\right],\left[\begin{array}{l}
4 \\
4 \\
1
\end{array}\right],\left[\begin{array}{l}
4 \\
1 \\
0
\end{array}\right],\left[\begin{array}{l}
1 \\
0 \\
0
\end{array}\right],\left[\begin{array}{l}
0 \\
0 \\
3
\end{array}\right],\left[\begin{array}{l}
0 \\
3 \\
2
\end{array}\right],\left[\begin{array}{l}
3 \\
3 \\
2
\end{array}\right],\left[\begin{array}{l}
3 \\
2 \\
0
\end{array}\right],\left[\begin{array}{l}
2 \\
0 \\
0
\end{array}\right]\right\}, \\
& H_{3}=\left\{\left[\begin{array}{l}
0 \\
1 \\
0
\end{array}\right],\left[\begin{array}{l}
1 \\
0 \\
3
\end{array}\right],\left[\begin{array}{l}
0 \\
3 \\
1
\end{array}\right],\left[\begin{array}{l}
3 \\
1 \\
0
\end{array}\right],\left[\begin{array}{l}
1 \\
0 \\
2
\end{array}\right],\left[\begin{array}{l}
0 \\
2 \\
0
\end{array}\right],\left[\begin{array}{l}
2 \\
0 \\
1
\end{array}\right],\left[\begin{array}{l}
0 \\
1 \\
2
\end{array}\right],\left[\begin{array}{l}
1 \\
2 \\
0
\end{array}\right],\left[\begin{array}{l}
2 \\
0 \\
4
\end{array}\right],\right. \\
& \left.\left[\begin{array}{l}
0 \\
4 \\
0
\end{array}\right],\left[\begin{array}{l}
4 \\
0 \\
2
\end{array}\right],\left[\begin{array}{l}
0 \\
2 \\
4
\end{array}\right],\left[\begin{array}{l}
2 \\
4 \\
0
\end{array}\right],\left[\begin{array}{l}
4 \\
0 \\
3
\end{array}\right],\left[\begin{array}{l}
0 \\
3 \\
0
\end{array}\right],\left[\begin{array}{l}
3 \\
0 \\
4
\end{array}\right],\left[\begin{array}{l}
0 \\
4 \\
3
\end{array}\right],\left[\begin{array}{l}
4 \\
3 \\
0
\end{array}\right],\left[\begin{array}{l}
3 \\
0 \\
1
\end{array}\right]\right\}, \\
& H_{4}=\left\{\left[\begin{array}{l}
0 \\
1 \\
3
\end{array}\right],\left[\begin{array}{l}
1 \\
3 \\
1
\end{array}\right],\left[\begin{array}{l}
3 \\
1 \\
3
\end{array}\right],\left[\begin{array}{l}
1 \\
3 \\
0
\end{array}\right],\left[\begin{array}{l}
3 \\
0 \\
2
\end{array}\right],\left[\begin{array}{l}
0 \\
2 \\
1
\end{array}\right],\left[\begin{array}{l}
2 \\
1 \\
2
\end{array}\right],\left[\begin{array}{l}
1 \\
2 \\
1
\end{array}\right],\left[\begin{array}{l}
2 \\
1 \\
0
\end{array}\right],\left[\begin{array}{l}
1 \\
0 \\
4
\end{array}\right],\right. \\
& \left.\left[\begin{array}{l}
0 \\
4 \\
2
\end{array}\right],\left[\begin{array}{l}
4 \\
2 \\
4
\end{array}\right],\left[\begin{array}{l}
2 \\
4 \\
2
\end{array}\right],\left[\begin{array}{l}
4 \\
2 \\
0
\end{array}\right],\left[\begin{array}{l}
2 \\
0 \\
3
\end{array}\right],\left[\begin{array}{l}
0 \\
3 \\
4
\end{array}\right],\left[\begin{array}{l}
3 \\
4 \\
3
\end{array}\right],\left[\begin{array}{l}
4 \\
3 \\
4
\end{array}\right],\left[\begin{array}{l}
3 \\
4 \\
0
\end{array}\right],\left[\begin{array}{l}
4 \\
0 \\
1
\end{array}\right]\right\}, \\
& H_{5}=\left\{\left[\begin{array}{l}
0 \\
1 \\
4
\end{array}\right],\left[\begin{array}{l}
1 \\
4 \\
2
\end{array}\right],\left[\begin{array}{l}
4 \\
2 \\
2
\end{array}\right],\left[\begin{array}{l}
2 \\
2 \\
0
\end{array}\right],\left[\begin{array}{l}
2 \\
0 \\
2
\end{array}\right],\left[\begin{array}{l}
0 \\
2 \\
3
\end{array}\right],\left[\begin{array}{l}
2 \\
3 \\
4
\end{array}\right],\left[\begin{array}{l}
3 \\
4 \\
4
\end{array}\right],\left[\begin{array}{l}
4 \\
4 \\
0
\end{array}\right],\left[\begin{array}{l}
4 \\
0 \\
4
\end{array}\right],\right. \\
& \left.\left[\begin{array}{l}
0 \\
4 \\
1
\end{array}\right],\left[\begin{array}{l}
4 \\
1 \\
3
\end{array}\right],\left[\begin{array}{l}
1 \\
3 \\
3
\end{array}\right],\left[\begin{array}{l}
3 \\
3 \\
0
\end{array}\right],\left[\begin{array}{l}
3 \\
0 \\
3
\end{array}\right],\left[\begin{array}{l}
0 \\
3 \\
2
\end{array}\right],\left[\begin{array}{l}
3 \\
2 \\
1
\end{array}\right],\left[\begin{array}{l}
2 \\
1 \\
1
\end{array}\right],\left[\begin{array}{l}
1 \\
1 \\
0
\end{array}\right],\left[\begin{array}{l}
1 \\
0 \\
1
\end{array}\right]\right\}, \\
& H_{6}=\left\{\left[\begin{array}{l}
1 \\
1 \\
1
\end{array}\right],\left[\begin{array}{l}
1 \\
1 \\
2
\end{array}\right],\left[\begin{array}{l}
1 \\
2 \\
3
\end{array}\right],\left[\begin{array}{l}
2 \\
3 \\
2
\end{array}\right],\left[\begin{array}{l}
3 \\
2 \\
2
\end{array}\right],\left[\begin{array}{l}
2 \\
2 \\
2
\end{array}\right],\left[\begin{array}{l}
2 \\
2 \\
4
\end{array}\right],\left[\begin{array}{l}
2 \\
4 \\
1
\end{array}\right],\left[\begin{array}{l}
4 \\
1 \\
4
\end{array}\right],\left[\begin{array}{l}
1 \\
4 \\
4
\end{array}\right],\right. \\
& \left.\left[\begin{array}{l}
4 \\
4 \\
4
\end{array}\right],\left[\begin{array}{l}
4 \\
4 \\
3
\end{array}\right],\left[\begin{array}{l}
4 \\
3 \\
2
\end{array}\right],\left[\begin{array}{l}
3 \\
2 \\
3
\end{array}\right],\left[\begin{array}{l}
2 \\
3 \\
3
\end{array}\right],\left[\begin{array}{l}
3 \\
3 \\
3
\end{array}\right],\left[\begin{array}{l}
3 \\
3 \\
1
\end{array}\right],\left[\begin{array}{l}
3 \\
1 \\
4
\end{array}\right],\left[\begin{array}{l}
1 \\
4 \\
1
\end{array}\right],\left[\begin{array}{l}
4 \\
1 \\
1
\end{array}\right]\right\},
\end{aligned}
$$




$$
\begin{gathered}
H_{7}=\left\{\left[\begin{array}{l}
1 \\
1 \\
3
\end{array}\right],\left[\begin{array}{l}
1 \\
3 \\
4
\end{array}\right],\left[\begin{array}{l}
3 \\
4 \\
1
\end{array}\right],\left[\begin{array}{l}
4 \\
1 \\
2
\end{array}\right],\left[\begin{array}{l}
1 \\
2 \\
2
\end{array}\right],\left[\begin{array}{l}
2 \\
2 \\
1
\end{array}\right],\left[\begin{array}{l}
2 \\
1 \\
3
\end{array}\right],\left[\begin{array}{l}
1 \\
3 \\
2
\end{array}\right],\left[\begin{array}{l}
3 \\
2 \\
4
\end{array}\right],\left[\begin{array}{l}
2 \\
4 \\
4
\end{array}\right]\right. \\
\left.\left[\begin{array}{l}
4 \\
4 \\
2
\end{array}\right],\left[\begin{array}{l}
4 \\
2 \\
1
\end{array}\right],\left[\begin{array}{l}
2 \\
1 \\
4
\end{array}\right],\left[\begin{array}{l}
1 \\
4 \\
3
\end{array}\right],\left[\begin{array}{l}
4 \\
3 \\
3
\end{array}\right],\left[\begin{array}{l}
3 \\
3 \\
4
\end{array}\right],\left[\begin{array}{l}
3 \\
4 \\
2
\end{array}\right],\left[\begin{array}{l}
4 \\
2 \\
3
\end{array}\right],\left[\begin{array}{l}
2 \\
3 \\
1
\end{array}\right],\left[\begin{array}{l}
3 \\
1 \\
1
\end{array}\right]\right\} \\
H_{8}=\left\{\left[\begin{array}{l}
1 \\
2 \\
4
\end{array}\right],\left[\begin{array}{l}
2 \\
4 \\
3
\end{array}\right],\left[\begin{array}{l}
4 \\
3 \\
1
\end{array}\right],\left[\begin{array}{l}
3 \\
1 \\
2
\end{array}\right]\right\}
\end{gathered}
$$

Note that the first row of $\mathrm{H}_{4}$ corresponds to the sequence in Example 1.1.

To further study the structure of the transitive $G$-sets, we turn to the eigenvalues and eigenvectors associated with the matrix $A$. By inspection, it is easy to see that the rank of $x I-A$ is $n$ or $n-1$. If $\lambda \in Z_{p}$ is a root of the characteristic polynomial $C(x)=\operatorname{det}(x I-A)=x^{n}-a_{n-1} x^{n-1}-$ $\cdots-a_{1} x-a_{0}$, then it can be verified that $\left[\begin{array}{c}1 \\ \lambda \\ \lambda^{2} \\ \cdot \\ \cdot \\ \cdot \\ \lambda^{n-1}\end{array}\right]$ is an eigenvector of $A$ and the dimension of the eigenspace corresponding to $\lambda$ is one. Write $E_{\lambda}$ for this eigenspace and note that

$$
E_{\lambda}=\left\{\left[\begin{array}{c}
0 \\
0 \\
0 \\
\cdot \\
\cdot \\
\cdot \\
0
\end{array}\right],\left[\begin{array}{c}
1 \\
\lambda \\
\lambda^{2} \\
\cdot \\
\cdot \\
\cdot \\
\lambda^{n-1}
\end{array}\right],\left[\begin{array}{c}
2 \\
2 \lambda \\
2 \lambda^{2} \\
\cdot \\
\cdot \\
\cdot \\
2 \lambda^{n-1}
\end{array}\right],\left[\begin{array}{c}
3 \\
3 \lambda \\
3 \lambda^{2} \\
\cdot \\
\cdot \\
\cdot \\
3 \lambda^{n-1}
\end{array}\right], \ldots,\left[\begin{array}{c}
(p-1) \\
(p-1) \lambda \\
(p-1) \lambda^{2} \\
\cdot \\
\cdot \\
\cdot \\
(p-1) \lambda^{n-1}
\end{array}\right]\right\}
$$

It is easy to check that $\left\{\left[\begin{array}{c}0 \\ 0 \\ \cdot \\ \cdot \\ \cdot \\ 0\end{array}\right]\right\}$ will always be a transitive $G$-set under the action of $G$ on $\left(Z_{p}\right)^{n}$. It is also easy to see that if a transitive $G$-set MISSOURI J. OF MATH. SCI., FALL 2014 


\section{T. MCKENZIE, S. OVERBAY, AND R. RAY}

contains an eigenvector, all the other vectors in that transitive $G$-set must also be eigenvectors. Therefore, for any transitive $G$-set, there are three mutually exclusive possibilities:

(1) it is the transitive $G$-set $\left\{\left[\begin{array}{l}0 \\ 0 \\ \cdot \\ \cdot \\ \cdot \\ 0\end{array}\right]\right\}$,

(2) it consists entirely of eigenvectors,

(3) it consists entirely of nonzero noneigenvectors.

In Example 2.1, the characteristic polynomial $C(x)=(x-2)^{3}$. So $\lambda=2$ is the only eigenvalue and the eigenspace is

$$
H_{1} \cup H_{8}=E_{2}=\left\{\left[\begin{array}{l}
0 \\
0 \\
0
\end{array}\right],\left[\begin{array}{l}
1 \\
2 \\
4
\end{array}\right],\left[\begin{array}{l}
2 \\
4 \\
3
\end{array}\right],\left[\begin{array}{l}
3 \\
1 \\
2
\end{array}\right],\left[\begin{array}{l}
4 \\
3 \\
1
\end{array}\right]\right\} \text {. }
$$

\section{The CAse $C(x)=(x-\lambda)^{n}$}

Throughout this section assume that

$$
C(x)=x^{n}-a_{n-1} x^{n-1}-\cdots-a_{1} x-a_{0}=(x-\lambda)^{n}
$$

for some $\lambda \in Z_{p}-\{0\}$.

Definition 3.1. Let $J$ be the elementary Jordan matrix associated with $C$ (see [3, p. 359]). So

$$
J=\left[\begin{array}{ccccc}
\lambda & 1 & 0 & \ldots & 0 \\
0 & \lambda & 1 & \ddots & 0 \\
0 & 0 & \lambda & \ddots & 0 \\
\vdots & \ddots & \ddots & \ddots & 1 \\
0 & 0 & 0 & \ldots & \lambda
\end{array}\right]
$$

Theorem 3.2. For all $i \in\{0,1, \ldots\}$,

$$
J^{i}=\left[\begin{array}{cccccc}
\lambda^{i} & \left(\begin{array}{c}
i \\
1
\end{array}\right) \lambda^{i-1} & \left(\begin{array}{c}
i \\
2 \\
2
\end{array}\right) \lambda^{i-2} & \left(\begin{array}{c}
i \\
3
\end{array}\right) \lambda^{i-3} & \ldots & \left(\begin{array}{c}
i \\
n-1
\end{array}\right) \lambda^{i-(n-1)} \\
0 & \lambda^{i} & \left(\begin{array}{c}
i \\
1
\end{array}\right) \lambda^{i-1} & \left(\begin{array}{c}
i \\
2
\end{array}\right) \lambda^{i-2} & \ldots & \left(\begin{array}{c}
n-2 \\
n-2
\end{array}\right) \lambda^{i-(n-2)} \\
0 & 0 & \lambda^{i} & \left(\begin{array}{c}
i \\
i \\
1
\end{array}\right) \lambda^{i-1} & \ldots & \left(\begin{array}{c}
i \\
n-3
\end{array}\right) \lambda^{i-(n-3)} \\
\vdots & \vdots & \vdots & \vdots & \vdots & \vdots \\
0 & 0 & 0 & 0 & \ldots & \lambda^{i}
\end{array}\right] .
$$




\section{JORDAN FORMS AND NTH ORDER LINEAR RECURRENCES}

In particular, if $k$ is the smallest power of $p$ such that $n \leq p^{k}$, then

$$
J^{\alpha p^{k}}=\left[\begin{array}{ccccc}
\lambda^{\alpha p^{k}} & 0 & 0 & \ldots & 0 \\
0 & \lambda^{\alpha p^{k}} & 0 & \ldots & 0 \\
0 & 0 & \lambda^{\alpha p^{k}} & \ldots & 0 \\
\vdots & \vdots & \vdots & \vdots & \vdots \\
0 & 0 & 0 & \ldots & \lambda^{\alpha p^{k}}
\end{array}\right]=\left[\begin{array}{ccccc}
\lambda^{\alpha} & 0 & 0 & \ldots & 0 \\
0 & \lambda^{\alpha} & 0 & \ldots & 0 \\
0 & 0 & \lambda^{\alpha} & \ldots & 0 \\
\vdots & \vdots & \vdots & \vdots & \vdots \\
0 & 0 & 0 & \ldots & \lambda^{\alpha}
\end{array}\right]
$$

and the order of $J$ is $p^{k}|\lambda|$, where $|\lambda|$ is the order of the unit $\lambda$ in the group $Z_{p}$.

Proof. The result follows from Lucas' Theorem [2], since if $i=p^{j}$, then $\left(\begin{array}{l}i \\ 1\end{array}\right),\left(\begin{array}{l}i \\ 2\end{array}\right),\left(\begin{array}{l}i \\ 3\end{array}\right), \ldots,\left(\begin{array}{c}i \\ n-1\end{array}\right)$, are all equal to 0 modulo $p$ if and only if $n \leq p^{j}$.

Theorem 3.3. Let $\mathbf{v} \in\left(Z_{p}\right)^{n}$. There are three mutually exclusive possibilities for the transitive $G$-set containing $\mathbf{v}$ :

(1) it is a transitive $G$-set of size one, consisting of only

(2) it consists entirely of eigenvectors of $A$ and has size $|\lambda|$,

(3) it consists entirely of nonzero noneigenvectors of $A$ and has size $p^{i}|\lambda|$, for some $i \in\{1, \ldots, k\}$, where $k$ is the smallest power of $p$ for which $n \leq p^{k}$.

Proof. Let

$$
G^{\prime}=\left\{J^{i}\left|0 \leq i \leq p^{k}\right| \lambda \mid-1\right\} .
$$

Since $J$ and $A$ are similar matrices, that is $A P=P J$ for some invertible matrix $P$, it is enough to show that the corresponding statement is true for the transitive $G^{\prime}$-set containing $\mathbf{w}=P^{-1}(\mathbf{v})$. Assume that $J^{i}(\mathbf{w})=\mathbf{w}$, for some positive integer $i$. Then $\mathbf{w}$ is an eigenvector of $J^{i}$ with eigenvalue 1 . By Theorem 3.2, the only eigenvalue of $J^{i}$ is $\lambda^{i}$. Thus, $\lambda^{i}=1$ and so $|\lambda|$ divides $i$.

In the first case, we have $\mathbf{v}=\mathbf{w}=\mathbf{0}$ so clearly the corresponding $G^{\prime}$-set has size one.

For the second case, assume $\mathbf{v}$ is an eigenvector of $A$, so $A \mathbf{v}=\lambda \mathbf{v}$. Then $P^{-1}(A \mathbf{v})=P^{-1}(\lambda \mathbf{v})$. Since $A$ and $J$ are similar, $J \mathbf{w}=\lambda \mathbf{w}$. Thus $\mathbf{w}$ is an eigenvector of $J$ with eigenvalue $\lambda$. This implies that $J^{|\lambda|} \mathbf{w}=\lambda^{|\lambda|} \mathbf{w}=\mathbf{w}$. So $J^{|\lambda|} \mathbf{w}=\mathbf{w}$ and no smaller positive power of $J$ can fix $\mathbf{w}$ because we know from the paragraph above that $|\lambda|$ would have to divide such a positive power.

MISSOURI J. OF MATH. SCI., FALL 2014 


\section{T. MCKENZIE, S. OVERBAY, AND R. RAY}

Finally, let $\mathbf{v}$ be a nonzero noneigenvector of $A$. By an argument similar to the one in the paragraph above, $\mathbf{w}$ is a nonzero noneigenvector of $J$. If $J^{i} \mathbf{w}=\mathbf{w}$, we know from the first paragraph that there exists $j \in\{1,2,3, \ldots\}$ such that $i=j|\lambda|$. By Theorem $3.2, J^{p^{k}|\lambda|}$ is the identity, so the size of the transitive $G^{\prime}$-set containing $\mathbf{w}$ divides $p^{k}|\lambda|$ (and has a factor of $|\lambda|$ ), in other words, $j$ divides $p^{k}$. Assume by way of contradiction that $j=1$. Applying Theorem 3.2 we see that

$$
J^{|\lambda|}=\left[\begin{array}{ccccc}
\lambda^{|\lambda|} & \left(\begin{array}{c}
|\lambda| \\
1
\end{array}\right) \lambda^{|\lambda|-1} & * & \ldots & * \\
0 & \lambda^{|\lambda|} & \left(\begin{array}{c}
|\lambda| \\
1
\end{array}\right) \lambda^{|\lambda|-1} & \ldots & * \\
0 & 0 & \lambda^{|\lambda|} & \ldots & * \\
\vdots & \vdots & \vdots & \vdots & \vdots \\
0 & 0 & 0 & \ldots & \lambda^{|\lambda|}
\end{array}\right]
$$

But this matrix equals

$$
\left[\begin{array}{ccccc}
1 & |\lambda| \lambda^{-1} & * & \ldots & * \\
0 & 1 & |\lambda| \lambda^{-1} & \ldots & * \\
0 & 0 & 1 & \ldots & * \\
\vdots & \vdots & \vdots & \vdots & \vdots \\
0 & 0 & 0 & \ldots & 1
\end{array}\right]
$$

Since $|\lambda| \lambda^{-1}$ is nonzero, it is easy to see that the null space of the matrix $J^{|\lambda|}-1 I$ is one dimensional. We have already shown that the eigenspace of $A$, namely $E_{\lambda}$ has cardinality $p$. So $P^{-1}\left(E_{\lambda}\right)$ is the complete one dimensional eigenspace of $J$ with eigenvalue $\lambda$. Thus, $P^{-1}\left(E_{\lambda}\right)$ is the complete one dimensional eigenspace of $J^{|\lambda|}$ with eigenvalue $\lambda^{|\lambda|}=1$. This contradicts the fact that $\mathbf{w}$ is a nonzero noneigenvector of $J$ with $J^{|\lambda|}(\mathbf{w})=\mathbf{w}$. Hence, $j$ does not equal 1 . This completes the proof.

We note that the $G$-sets corresponding to nonzero noneigenvectors may have different sizes. Let $C(x)=(x-2)^{5}$ and

$$
A=\left[\begin{array}{lllll}
0 & 1 & 0 & 0 & 0 \\
0 & 0 & 1 & 0 & 0 \\
0 & 0 & 0 & 1 & 0 \\
0 & 0 & 0 & 0 & 1 \\
2 & 1 & 2 & 2 & 1
\end{array}\right]
$$




\section{JORDAN FORMS AND NTH ORDER LINEAR RECURRENCES}

taken over $Z_{3}$. The $G$-set associated with $\left[\begin{array}{l}0 \\ 1 \\ 0 \\ 0 \\ 2\end{array}\right]$ has size $6=3^{1} \cdot 2$, while the $G$-set associated with $\left[\begin{array}{l}0 \\ 2 \\ 1 \\ 0 \\ 0\end{array}\right]$ has size $18=3^{2} \cdot 2$.

Theorem 3.4. Let $\mathbf{w} \in\left(Z_{p}\right)^{n}$. Then for all $i, \alpha \in\{0,1, \ldots\}$,

$$
J^{i+\alpha p^{k}}(\mathbf{w})=\lambda^{\alpha} \cdot J^{i}(\mathbf{w}),
$$

where $k$ is the smallest power of $p$ for which $n \leq p^{k}$.

Proof. By Theorem 3.2, $J^{\alpha p^{k}}(\mathbf{w})=\lambda^{\alpha} \mathbf{w}$. Thus, $J^{i+\alpha p^{k}}(\mathbf{w})=J^{i} J^{\alpha p^{k}}(\mathbf{w})=$ $J^{i}\left(\lambda^{\alpha}(\mathbf{w})\right)=\lambda^{\alpha} \cdot J^{i}(\mathbf{w})$.

Definition 3.5. If $\mathbf{v}=\left[\begin{array}{c}v_{1} \\ v_{2} \\ \vdots \\ v_{n}\end{array}\right]$ is a vector, then $\pi_{1}(\mathbf{v})=v_{1}$.

By examining the Jordan form $J$ of $A$, we have obtained results for the sizes of the $G^{\prime}$-sets corresponding to $J$. This gives us information about cardinality of the $G$-sets corresponding to $A$ and periods of the related sequences. These results hold for any invertible matrix $P$ with $A P=$ $P J$. Next we will construct a specific matrix $P$, with the property that $\pi_{1}\left(A^{i} \mathbf{v}\right)=\pi_{1}\left(J^{i}\left(P^{-1} \mathbf{v}\right)\right)$, for all $i \in\{0,1, \ldots\}$.

Lemma 3.6. Given $i \in\{1, \ldots, n-1\}$, write $C^{(i)}(x)$ or $\frac{d^{i}}{d x^{i}} C(x)$ for the ith (formal) derivative of the polynomial $C(x)$. Then $C^{(i)}(x)$ evaluated at $\lambda$ equals zero.

Proof. See Lemma 6.10, [3, p. 161].

Lemma 3.7. Given $i \in\{1, \ldots, n-1\},\left.\frac{1}{i !} \cdot \frac{d^{i}}{d x^{i}}\right|_{x=\lambda}\left(x^{n}-C(x)\right)=\left(\begin{array}{c}n \\ i\end{array}\right) \lambda^{n-i}$.

Proof. By the previous lemma, we know that $C^{(i)}(\lambda)=\left.\frac{d^{i}}{d x^{i}}\right|_{x=\lambda} C(x)=0$. 
Thus,

$$
\left.\frac{1}{i !} \cdot \frac{d^{i}}{d x^{i}}\right|_{x=\lambda}\left(x^{n}-C(x)\right)=\left.\frac{1}{i !} \cdot \frac{d^{i}}{d x^{i}}\right|_{x=\lambda} x^{n}=\frac{1}{i !} \frac{n !}{(n-i) !} \lambda^{n-i}=\left(\begin{array}{c}
n \\
i
\end{array}\right) \lambda^{n-i} .
$$

For the remainder of this section we will use a specific invertible matrix $P$.

Definition 3.8. Let $P=\left[p_{i j}\right]$ be the $n \times n$ matrix

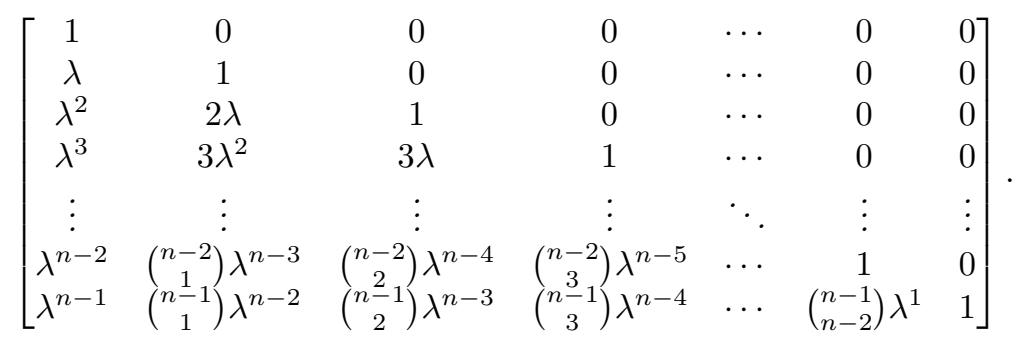

Here, $p_{i j}=\left(\begin{array}{c}i-1 \\ j-1\end{array}\right) \lambda^{i-j}$.

Lemma 3.9. $P$ is invertible and $P^{-1}=$

$$
\left[\begin{array}{cccccc}
1 & 0 & 0 & \cdots & 0 & 0 \\
-\lambda & 1 & 0 & \cdots & 0 & 0 \\
\lambda^{2} & -2 \lambda & 1 & \cdots & 0 & 0 \\
-\lambda^{3} & 3 \lambda^{2} & -3 \lambda & \cdots & 0 & 0 \\
\vdots & \vdots & \vdots & \ddots & \vdots & \vdots \\
(-\lambda)^{n-2} & \left(\begin{array}{c}
n-2 \\
1
\end{array}\right)(-\lambda)^{n-3} & \left(\begin{array}{c}
n-2 \\
2
\end{array}\right)(-\lambda)^{n-4} & \cdots & 1 & 0 \\
(-\lambda)^{n-1} & \left(\begin{array}{c}
n-1 \\
1
\end{array}\right)(-\lambda)^{n-2} & \left(\begin{array}{c}
n-1 \\
2
\end{array}\right)(-\lambda)^{n-3} & \cdots & \left(\begin{array}{c}
n-1 \\
n-2
\end{array}\right)(-\lambda)^{1} & 1
\end{array}\right] .
$$

Proof. This is easily checked.

Theorem 3.10. $A P=P J$.

Proof. First we consider the product $P J$. If $i \in\{1, \ldots, n\}$, then the dot product of the $i$ th row of $P$ and the first column of $J$ is $\lambda^{i}$. If $j \in\{2, \ldots, n\}$, then the dot product of the $i$ th row of $P$ and the $j$ th column of $J$ is

$$
\lambda \cdot\left(\begin{array}{l}
i-1 \\
j-1
\end{array}\right) \lambda^{i-j}+\left(\begin{array}{l}
i-1 \\
j-2
\end{array}\right) \lambda^{i-j+1}=\left(\left(\begin{array}{l}
i-1 \\
j-1
\end{array}\right)+\left(\begin{array}{l}
i-1 \\
j-2
\end{array}\right)\right) \cdot \lambda^{i-j+1} .
$$




\section{JORDAN FORMS AND NTH ORDER LINEAR RECURRENCES}

By Pascal's Identity, this reduces to $\left(\begin{array}{c}i \\ j-1\end{array}\right) \lambda^{i-j+1}$. So

$$
P J=\left[\begin{array}{ccccccc}
\lambda & 1 & 0 & 0 & \ldots & 0 & 0 \\
\lambda^{2} & 2 \lambda & 1 & 0 & \ldots & 0 & 0 \\
\lambda^{3} & 3 \lambda^{2} & 3 \lambda & 1 & \ldots & 0 & 0 \\
\lambda^{4} & 4 \lambda^{3} & 6 \lambda^{2} & 4 \lambda & \ldots & 0 & 0 \\
\vdots & \vdots & \vdots & \vdots & \ldots & 0 & 0 \\
\lambda^{n-1} & \left(\begin{array}{c}
n-1 \\
1
\end{array}\right) \lambda^{n-2} & \left(\begin{array}{c}
n-1 \\
2
\end{array}\right) \lambda^{n-3} & \left(\begin{array}{c}
n-1 \\
3
\end{array}\right) \lambda^{n-4} & \ldots & 1 & 0 \\
\lambda^{n} & \left(\begin{array}{c}
n \\
1
\end{array}\right) \lambda^{n-1} & \left(\begin{array}{c}
n \\
2
\end{array}\right) \lambda^{n-2} & \left(\begin{array}{c}
n \\
3
\end{array}\right) \lambda^{n-3} & \ldots & \left(\begin{array}{c}
n \\
n-2
\end{array}\right) \lambda^{2} & n \lambda
\end{array}\right] .
$$

Next we consider the product $A P$. If $i \in\{1, \ldots, n-1\}$ and $j \in\{1, \ldots, n\}$, then the dot product of the $i$ th row of $A$ and the $j$ th column of $P$ is the $i+1, j$ th entry of $P$, i.e. $\left(\begin{array}{c}i \\ j-1\end{array}\right) \lambda^{i-j+1}$. So

$A P=$

$$
\left[\begin{array}{cccccccc}
\lambda & 1 & 0 & 0 & 0 & \ldots & 0 & 0 \\
\lambda^{2} & 2 \lambda & 1 & 0 & 0 & \ldots & 0 & 0 \\
\lambda^{3} & 3 \lambda^{2} & 3 \lambda & 1 & 0 & \ldots & 0 & 0 \\
\lambda^{4} & 4 \lambda^{3} & 6 \lambda^{2} & 4 \lambda & 1 & \ldots & 0 & 0 \\
\vdots & \vdots & \vdots & \vdots & \vdots & \ldots & 0 & 0 \\
\lambda^{n-1} & \left(\begin{array}{c}
n-1 \\
1
\end{array}\right) \lambda^{n-2} & \left(\begin{array}{c}
n-1 \\
2
\end{array}\right) \lambda^{n-3} & \left(\begin{array}{c}
n-1 \\
3
\end{array}\right) \lambda^{n-4} & \left(\begin{array}{c}
n-1 \\
4
\end{array}\right) \lambda^{n-5} & \ldots & 1 & 0 \\
* & * & * & * & * & \ldots & * & *
\end{array}\right] .
$$

All that remains is to show that the last row of $A P$ and $P J$ are equal. The dot product of the $n$th row of $A$ and the $j$ th column of $P$ is

$$
\begin{aligned}
& a_{j-1} p_{j j}+\cdots+a_{n-1} p_{n j} \\
& =a_{j-1}\left(\begin{array}{c}
j-1 \\
j-1
\end{array}\right) \lambda^{0}+a_{j}\left(\begin{array}{c}
j \\
j-1
\end{array}\right) \lambda^{1}+\cdots+a_{n-1}\left(\begin{array}{c}
n-1 \\
j-1
\end{array}\right) \lambda^{n-j} \\
& =\frac{1}{(j-1) !} \cdot\left(a_{j-1} \frac{(j-1) !}{0 !} \lambda^{0}+a_{j} \frac{(j) !}{1 !} \lambda^{1}+\cdots+a_{n-1} \frac{(n-1) !}{(n-j) !} \lambda^{n-j}\right) \\
& =\left.\frac{1}{(j-1) !} \cdot \frac{d^{j-1}}{d x^{j-1}}\right|_{x=\lambda}\left(a_{0}+a_{1} x^{1}+a_{2} x^{2}+\cdots+a_{n-1} x^{n-1}\right) \\
& =\left.\frac{1}{(j-1) !} \cdot \frac{d^{j-1}}{d x^{j-1}}\right|_{x=\lambda}\left(x^{n}-C(x)\right) .
\end{aligned}
$$

But by Lemma 3.7, this equals $\left(\begin{array}{c}n \\ j-1\end{array}\right) \lambda^{n-j+1}$. This is precisely the entry in the last row, $j$ th column of $P J$. Thus, $A P=P J$.

Theorem 3.11. For all $i \in\{0,1, \ldots\}$ and $\mathbf{v} \in\left(Z_{p}\right)^{n}, J^{i}\left(P^{-1} \mathbf{v}\right)=P^{-1}\left(A^{i} \mathbf{v}\right)$ and $\pi_{1}\left(A^{i} \mathbf{v}\right)=\pi_{1}\left(J^{i}\left(P^{-1} \mathbf{v}\right)\right)$. 


\section{T. MCKENZIE, S. OVERBAY, AND R. RAY}

Proof. By Theorem 3.10,

$$
\begin{aligned}
J^{i}\left(P^{-1} \mathbf{v}\right) & \left.=\left(P^{-1} A P\right)^{i}\left(P^{-1} \mathbf{v}\right)\right) \\
& =\left(P^{-1} A^{i} P\right)\left(P^{-1} \mathbf{v}\right) \\
& =P^{-1}\left(A^{i} \mathbf{v}\right)
\end{aligned}
$$

By Lemma 3.9 , the first row of $P^{-1}$ is $(1,0,0, \ldots, 0)$, so clearly $\pi_{1}\left(A^{i} \mathbf{v}\right)=$ $\pi_{1}\left(P^{-1}\left(A^{i} \mathbf{v}\right)\right)$.

Corollary 3.12. As mentioned earlier, a sequence $\left\{S_{i}\right\}$ that is generated by $A$ can be described by $S_{i}=\pi_{1}\left(A^{i} \mathbf{v}\right)$, for some $\mathbf{v} \in\left(Z_{p}\right)^{n}$. Consequently, $\left\{S_{i}\right\}$ is uniformly distributed if and only if the elements $\pi_{1}\left(J^{i}\left(P^{-1} \mathbf{v}\right)\right)$ are uniformly distributed.

Example 3.13. If $A$ is the matrix from Example 2.1, then $\lambda=2, J=$ $\left[\begin{array}{lll}2 & 1 & 0 \\ 0 & 2 & 1 \\ 0 & 0 & 2\end{array}\right], P=\left[\begin{array}{lll}1 & 0 & 0 \\ 2 & 1 & 0 \\ 4 & 4 & 1\end{array}\right]$, and $P^{-1}=\left[\begin{array}{lll}1 & 0 & 0 \\ 3 & 1 & 0 \\ 4 & 1 & 1\end{array}\right]$. Now let $\mathbf{w}=$ $P^{-1}(\mathbf{v})$, where $\mathbf{v}=\left[\begin{array}{l}0 \\ 1 \\ 3\end{array}\right]$, the initial conditions of the sequence in Example 1.1. The $G^{\prime}$-set corresponding to $\mathbf{w}$ is

$$
\begin{gathered}
K=\left\{\left[\begin{array}{l}
0 \\
1 \\
4
\end{array}\right],\left[\begin{array}{l}
1 \\
1 \\
3
\end{array}\right],\left[\begin{array}{l}
3 \\
0 \\
1
\end{array}\right],\left[\begin{array}{l}
1 \\
1 \\
2
\end{array}\right],\left[\begin{array}{l}
3 \\
4 \\
4
\end{array}\right],\left[\begin{array}{l}
0 \\
2 \\
3
\end{array}\right],\left[\begin{array}{l}
2 \\
2 \\
1
\end{array}\right],\left[\begin{array}{l}
1 \\
0 \\
2
\end{array}\right],\left[\begin{array}{l}
2 \\
2 \\
4
\end{array}\right],\left[\begin{array}{l}
1 \\
3 \\
3
\end{array}\right],\right. \\
\left.\left[\begin{array}{l}
0 \\
4 \\
1
\end{array}\right],\left[\begin{array}{l}
4 \\
4 \\
2
\end{array}\right],\left[\begin{array}{l}
2 \\
0 \\
4
\end{array}\right],\left[\begin{array}{l}
4 \\
4 \\
3
\end{array}\right],\left[\begin{array}{l}
2 \\
1 \\
1
\end{array}\right],\left[\begin{array}{l}
0 \\
3 \\
2
\end{array}\right],\left[\begin{array}{l}
3 \\
3 \\
4
\end{array}\right],\left[\begin{array}{l}
4 \\
0 \\
3
\end{array}\right],\left[\begin{array}{l}
3 \\
3 \\
1
\end{array}\right],\left[\begin{array}{l}
4 \\
2 \\
2
\end{array}\right]\right\} .
\end{gathered}
$$

Note that the sequence in Example 1.1 is the first row of both $K$ and $H_{4}$ of Example 2.1.

Finally, we observe that Theorem 3.2 can be generalized to the case where

$$
C(x)=\left(x-\lambda_{1}\right)^{n_{1}}\left(x-\lambda_{2}\right)^{n_{2}} \cdots\left(x-\lambda_{t}\right)^{n_{t}}
$$

over $Z_{p}$. The corresponding Jordan forms will consist of blocks of elementary Jordan matrices (see Definition 3.1) for each $\lambda_{i}$. Applying Theorem 3.2 to each block, we see that the order of $A$ is equal to the least common multiple of the orders of the elementary Jordan blocks. The distribution properties for second order recurrences over $Z_{p}$ are well-known [5]. As seen in Example 2.1, not all $G$-sets corresponding to nonzero noneigenvectors are uniformly distributed. A general characterization of the distribution properties of third order recurrences appears in [4] and partial results for higher order linear recurrences are discussed in [7]. To the best of our 


\section{JORDAN FORMS AND NTH ORDER LINEAR RECURRENCES}

knowledge, general criteria for the distribution properties of $n$th order linear recurrences are not known for large $n$.

\section{REFERENCES}

[1] M. Artin, Algebra, Prentice Hall, New Jersey, 1991.

[2] N. J. Fine, Binomial coefficients modulo a prime, The American Mathematical Monthly, 54.10, Part 1 (1947), 589-592.

[3] T. Hungerford, Algebra, Springer, New York, 1974.

[4] M. J. Knight and W. A. Webb, Uniform distribution of third order linear recurrence sequences, Acta Arithmetica, 36 (1980), 6-20.

[5] R. Lidl and H. Niederreiter, Introduction to Finite Fields and Their Applications, Cambridge University Press, Cambridge, 1986.

[6] T. McKenzie, S. Overbay, and R. Ray, G-sets and linear recurrences modulo primes, Missouri Journal of Mathematical Sciences, 21.1 (2013), 27-36.

[7] H. Niederreiter and J.-S. Shiue, Equidistribution of linear recurring sequences in finite fields, Indagationes Mathematicae, 80 (1977), 397-405.

MSC2010: 11B50

Key words and phrases: matrix groups, linear recurrences over $Z_{p}$

Department of Mathematics, Gonzaga University, Spokane, WA

E-mail address: mckenzie@gonzaga.edu

Department of Mathematics, Gonzaga University, Spokane, WA

E-mail address: overbay@gonzaga.edu

Department of Mathematics, Gonzaga University, Spokane, WA

E-mail address: rayr@gonzaga.edu 